\title{
Treating depression in HIV/AIDS
}

M Y H Moosa, MMed (Psych), FCPsych, MBChB, MCFP, BSC

F Y Jeenah, MMed (Psych), FCPsych, MB ChB

Department of Neurosciences, Division of Psychiatry, University of the Witwatersrand, Johannesburg

The prevalence of HIV/AIDS has reached alarming proportions in South Africa. Although it is strongly associated with depressive moods, there are very few published studies on its treatment in patients with HIV/AIDS. This article reviews the prevalence, treatment and potential effects of depressive disorders on immunity and adherence to antiretroviral therapy (ART).

The studied prevalence of depressive disorders in HIV-positive patients varies widely, ranging from $0 \%$ to $47.8 \%$. However, these patients have nearly twice the likelihood of having had a recent episode of major depressive disorder compared with HIV-negative individuals.

Currently available antidepressant medications are equally effective in treating HIV/AIDS patients and the general population. Furthermore, intervention studies have shown that psychotherapy reduces depressive symptoms and is well tolerated. Interpersonal psychotherapy is more successful than supportive psychotherapy in lessening depression, and patients experience improved functioning physically and emotionally.

Untreated depression may be associated with reduced adherence to ART, immunosuppression, and more rapid HIV illness progression. In South Africa, HIV/AIDS patients may be at greater risk for psychiatric disorder given the potentially stressful living conditions including high rates of unemployment and poverty, poor and unstable housing, inadequate social services, and high rates of crime and domestic violence. A lack of data on depression in South Africa underscores the need for further research.

HIV/AIDS has reached pandemic proportions in South Africa. The National HIV and Syphilis Seroprevalence Survey' revealed that in 2004 there were about 5.3 million people living with HIV/AIDS in South Africa; $26.5 \%$ of all pregnant women tested positive for HIV, and there were more than 1500 new infections daily. Worldwide, UNAIDS estimated that at the end of 2003, $1.1 \%$ of adults between the ages of 15 and 49 years were living with HIV/AIDS, 3 million adults and children had died of AIDS, and 15 million children had been orphaned. ${ }^{2}$

\section{Prevalence of depression}

Worldwide, depression is exceedingly common in the general population, with lifetime prevalence rates ranging from 9\% to $20 \%{ }^{3}$ Depression is twice as common in women $(20 \%)$ compared with men (10\%). ${ }^{4}$ In patients with chronic medical diseases the rates are higher, at $15-36 \% .^{5}$ Depression also appears to occur frequently among HIV/AIDS patients, emerging soon after diagnosis or during the course of illness, with major depressive disorder being the most common disorder. ${ }^{6}$

Being diagnosed with a stigmatising disorder, experience of severe physical symptoms, and loss of relationships or work as a result of the diagnosis may all contribute to the development of HIV/AIDS-associated depression. ' Depression may also be secondary to neuropathology caused by HIV/AIDS itself. ${ }^{8}$ The distinction between major depressive disorder and mood disorder due to HIV disease can be difficult, since both result in similar symptoms. Fatigue, lethargy, low libido, diminished appetite and weight loss may be manifestations of either HIV-related illnesses or depressive disorder. In contrast, cognitive symptoms, feeling sad, losing interest in formerly enjoyable activities, guilt, and irritability are usually aspects of mood affliction.

The prevalence of depressive disorders in HIV-positive patients varies widely in the literature, ranging from $0 \%$ to $47.8 \%$. $6,9-11$ In clinical samples the rates range from $2 \%$ to $35 \%, 12,13$ while rates vary from $30 \%$ to $60 \%$ in community samples. ${ }^{14,15}$ Yet other studies that compared rates of depressive disorders in HIV-positive and negative patients (matched for gender, sexual orientation and drug use) concluded that HIV infection is not associated with a higher rate of the disorder. ${ }^{16,17}$ However, a meta-analysis of some of these published studies by Ciesla et al. ${ }^{18}$ concluded that HIV-positive individuals have nearly twice the likelihood of having had a recent episode of major depressive disorder compared with HIV-negative individuals. This is probably a better estimate, as the study considered differences between groups for a number of potentially important factors such as gender, mode of transmission, access to quality health care, socio-economic status and advances in the treatment of HIV infection.

\section{Treatment of depression}

\section{Pharmacological treatments}

Treatment of depression in HIV-positive patients has received little systematic study, ${ }^{19}$ even though medication tolerance and 
potential medication interactions in such patients may influence the effectiveness and safety of antidepressant medication. ${ }^{20}$

Double-blind, randomised, placebo-controlled trials of imipramine, $^{21,22}$ fluoxetine $^{23}$ and paroxetine ${ }^{24}$ have shown response rates ranging from $45 \%$ to $80 \%$, with a placebo response of up to $48 \%$. Despite these significant effects of medication, attrition rates were as high as $55 \%$. By the end of 6 months more than one-third of responders had discontinued imipramine because of troublesome anticholinergic side-effects (such as dry mouth, fatigue, and muscle aches). Although tricyclic antidepressants produced significant response rates, adverse effects limit their usefulness. Selective serotonin re-uptake inhibitors (SSRIs), although not more efficacious, are more tolerable and have greater overall effectiveness. ${ }^{25}$ In addition to the absence of anticholinergic side-effects, SSRIs are safer in overdose than the tricyclic antidepressants, which is an advantage in HIV patients given their higher risk of suicidal ideation. ${ }^{26}$

\section{Psychotherapy treatments}

Psychotherapy intervention studies using large samples with sufficient statistical power have shown that psychotherapy reduces depressive symptoms, is well tolerated by HIV-infected individuals, and resulted in significant changes in the immunity of responders. ${ }^{27,28}$ Markowitz et al. ${ }^{29}$ have shown that interpersonal psychotherapy (IPT) was successful in resolving the depression of $87 \%$ of their sample. They found that in the case of depressed HIVpositive patients who were not acutely ill, IPT was more successful than supportive psychotherapy in alleviating depression. Differences were observable by the middle of treatment ( 8 weeks) and prevailed to termination at 12 weeks. Patients receiving IPT experienced both increased physical functioning lactivities like returning to work, socialising, etc.) and emotional improvement.

Further psychotherapy treatment trials employing specific immune measures of relevance to HIV disease progression and including follow-up assessments of mood, immunity and disease progression will be helpful in drawing conclusions on the definitive role of these treatments in HIV/AIDS-related depression.

\section{Untreated depression}

Studies of depressed but otherwise medically healthy individuals have failed to demonstrate an overall effect on the immune system. ${ }^{30,31}$ There is also a paucity of research on alterations in immunity among HIV-positive individuals who are depressed. The association between depression and immunosuppression, 32 as well as between depression and more rapid HIV illness progression ${ }^{33}$ suggest that treating depression may have a positive effect on measures of immune status. Conversely, in treating patients with vulnerable immune systems there is concern that medication may have an adverse negative immunosuppressive effect. Although neither effect was observed in studies on tricyclic antidepressant medications, no data are available on SSRIs.

Untreated depression is associated with reduced adherence to antiretroviral therapy (ART). Adherence is the key to the effectiveness of ART. ${ }^{34}$ Non-adherence may lead to ART failure and the development of medication resistance, which may worsen prognosis and limit future treatment options. ${ }^{35,36}$ To ensure that the viral load in a patient is kept at undetectable levels, an adherence rate of between $90 \%$ and $100 \%$ is required. . $^{37,38}$ The nature of ART regimens makes adherence particularly difficult, as with complex regimens many pills are taken during a day at different times, some with food and some without. ART has unpleasant side-effects including diarrhoea, nausea, vomiting and peripheral neuropathy.

It has been shown that antidepressant treatment improves adherence to ART in depressed HIV-positive patients. ${ }^{9}$ ART adherence improved in the 6 months following the prescription of antidepressant therapy regardless of the patient's preceding levels of adherence. Adherence to ART was highest in patients who were also adherent to their antidepressant medication.

\section{Conclusion}

The importance of the assessment and treatment of depression in individuals with HIV/AIDS is underscored by the decreased adherence to medication regimens in the absence thereof, and by a potential further suppression of immunity and accelerated disease progression. Available data show that currently available treatments are effective for treating depressive disorders in patients with HIV/AIDS. However, much of the work on psychiatric morbidity in HIV/AIDS has been done in the West. In South Africa, HIV/AIDS patients may be at greater risk for psychopathology than patients in certain parts of the developed world given the potentially stressful living conditions including high rates of unemployment and poverty, poor and unstable housing, inadequate social services, and high rates of crime and domestic violence. As such screening, evaluation and treatment of these disorders is crucial and should be done routinely.

\section{References}

1. National HIV and Syphilis Antenatal Seroprevalence Survey in South Africa 2004 Pretoria: Department of Health, 2005. http://www.doh.gov.za/docs/ llast accessed 14 June 2006).

2. UNAIDS and World Health Organization (WHO). AIDS Epidemic Update: December 
2004. Geneva: UNAIDS/WHO, 2004: 1

3. Barry KL, Fleming MF, Manwell LB, et al. Prevalence of and factors associated with current and lifetime depression in older adult primary care patients. Fam Med 1998 30: $366-371$

4. Steffens DC, Skoog I, Norton MC, et al. Prevalence of depression and its treatment in an elderly population: The Cache County study. Arch Gen Psychiatry 2000; 57 : $601-607$

5. Feldman E, Mayou R, Hawton K, et al. Psychiatric disorder in medical in-patients. QJ Med 1987; 63: 405-412

6. Olley BO, Seedat S, Stein DJ. Persistence of psychiatric disorders in a cohort of HIV/ AIDS patients in South Africa: a 6-month follow-up study. J Psychosom Res 2006; 61: $479-484$

7. Zibler C. Psychotherapeutic strategies for coping with HIV infection. In: Cournos F Forstein M, eds. What Mental Health Practifioners Need to Know About HIV and AIDS. San Francisco: Jossey-Bass, 2000: 5 1-56.

8. Maj $M$, Janssen R, Starace $F$, et al. WHO neuropsychiatric AIDS study, cross-sectional phase I: study design and psychiatric findings. Arch Gen Psychiatry 1994; 51 : 3949

9. Yun LWH, Maravi, M., Kobayashi, et al. Antidepressant treatment improves adherence to antiretroviral therapy among depressed HIV-infected patients. I Acquir Immune Defic Syndr 2005; 38: 432-438

10. Maj M. Depressive syndromes and symptoms in subjects with human immunodeficiency virus (HIV) infection. BrJ Psychiatry 1996; 30: 117-122.

11. Lyketsos CG, Hanson A, Fishman M, et al. Screening for psychiatric morbidity in a medical outpatient clinic for HIV infection: the need for a psychiatric presence. IntJ Psychiatry Med 1994; 24: 103-1 13

12. Goggin K, Engelson ES, Rabkin JG, et al. The relationship of mood, endocrine, and sexual disorders in human immunodeficiency virus positive $(\mathrm{HIV}+)$ women: an exploratory study. Psychosom Med 1998; 60: 11-16.

13. Taylor ER, Amodei N, Mangos R. The presence of psychiatric disorders in HIV-infected women. Journal of Counseling and Development 1996; 74: 345-351

14. Ickovics JR, Hamburger ME, Vlahov D, et al. Mortality, CD4 cell count decline, and depressive symptoms among HIV-seropositive women. JAMA 200 1; 285: 1466. 1474

15. Moore J, Schuman P, Schoenbaum E, et al. Severe adverse life events and depressive symptoms among women with, or at risk for, HIV infection in four cities in the United States of America. AlDS 1999: 13: 2459-2468.

16. Fukunishi I, Matsumoto T, Negishi M, et al. Somatic complaints associated with depressive symptoms in HIV-positive patients. Psychother Psychosom 1997; 66: 248 251.

17. Dew MA, Becker JT, Sanchez J, et al. Prevalence and predictors of depressive, anxiety, and substance use disorders in HIV-infected and uninfected men: a longitudinal evaluation. Psychol Med 1997; 27: 395-409.

18. Ciesla JA, Roberts JE. Meta-analysis of the relationship between HIV infection and risk for depressive disorders. Am J Psychiatry 2001; 1 58: 725-730.
19. Gill D. Do antidepressants work in depression in physical illness? Proceedings of Academy of Psychosomatic Medicine 44th Annual Meeting, Chicago, 6-10 November 1997: 37-38.

20. Elliott AJ, Roy-Byrne PP. Major depressive disorder and HIV-1 infection: A review of treatment trials. Semin Clin Neuropsychiatry 1998; 3: 137-150.

21. Markowitz J, Kocsis JH, Fishman B, et al. Treatment of depressive symptoms in HIVpositive patients. Arch Gen Psychiatry 1998; 55: 452-457.

22. Rabkin JG, Wagner $G$, Rabkin R. Effects of sertraline on mood and immune status in patients with depression and HIV illness. J Clin Psychiatry 1994; 55: 433-439.

23. Zisook S, Peterkin J, Goggin KJ, et al. Treatment of major depression in HIV-seropositive men. J Clin Psychiatry 1998; 59: 217-224.

24. Elliott AJ, Uldall KK, Bergam K, et al. Randomized, placebo-controlled trial of paroxetine versus imipramine in depressed HIV-positive outpatients. Am J Psychiatry 1998; 155: 367-372.

25. Penzak SR, Reddy YS, Grimsley SR. Depression in patients with HIV infection. Am J Health Syst Pharm 2000; 57: 376-386.

26. Starace F. Epidemiology of suicide among persons with AIDS. AIDS Care 1995; 7 : S123-S128.

27. Goodkin K, Feaster DJ, Asthana D, et al. A bereavement support group intervention is longitudinally associated with salutary effects on the CD4 cell count and number of physician visits. Clin Diagn Lab Immunol 1998; 5: 382-391

28. Gregerson MB, Roberts IM, Amiri MM. Absorption and imagery locate immune response in the body. Biofeedback \& Self Regulation 1996; 21: 149-165.

29. Markowitz JC, Klerman GL, Perry SW. Interpersonal psychotherapy of depressed HIV positive outpatients. Hospital \& Community Psychiatry 1992; 43: 885-890.

30. Schleifer SJ, Keller SE, Bartlett JA. Depression and immunity: clinical factors and therapeutic course. Psychiatry Res 1999; 85: 63-69

31. Frank MG, Hendricks SE, Johnson DR, et al. Antidepressants augment natural killer cell activity: in vivo and in vitro. Neuropsychobiology 1997; 39: 18-24

32. Stein $M$, Miller A, Treisman R. Depression, the immune system, and health and illness: findings in search of meaning. Arch Gen Psychiatry 1991; 48: 171-177.

33. Mayne TJ, Vittinghoff $E$, Chesney $M$, et al. Depressive affect and survival among gay and bisexual men infected with HIV. Arch Intern Med 1996; 156: 2233-2238.

34. Carpenter CC, Fischl MA, Hammer SM, et al. Antiretroviral therapy for HIV infection in 1998. Updated recommendations of the International AIDS Society - USA Panel. IAMA 1998; 280: 78-86

35. Stephenson J. AIDS researchers target poor adherence. JAMA 1999; 281:1069

36. Wainberg MA. Friedlang $G$. Public health implications of anti-retroviral therapy and HIV drug resistance. JAMA 1998; 279; 1977-1983.

37. Paterson D, Swindells S, Morh J, et al. Adherence to protease inhibitor therapy and outcomes in patients with HIV infection. Ann Intern Med 2000; 133: 21-30.

38. Singh N, Berman SM, Swindells $S$, et al. Adherence of human immunodeficiency virusinfected patients to antiretroviral therapy. Clin Infect Dis 1999; 29: 824-830. 